\title{
Correction to: Liaoning Score for Prediction of Esophageal Varices in Cirrhotic Patients Who Had Never Undergone Endoscopy: A Multicenter Cross- Sectional Study in Liaoning Province, China
}

\author{
Xingshun Qi (1) - Yiling Li - Ran Wang - Lianjie Lin · Jing Li · \\ Lijun Wang · Shuang Zheng · Yonghong Sun · Lixin Zhao · \\ Xiaolin Fu $\cdot$ Mengchun Wang $\cdot$ Xinping Qiu $\cdot$ Han Deng · \\ Cen Hong · Qianqian Li · Hongyu Li · Xiaozhong Guo
}

Published online: June 24, 2019

( ) Springer Healthcare Ltd., part of Springer Nature 2019

Correction to: Adv Ther

https://doi.org/10.1007/s12325-019-00967-w

Unfortunately, the fourteenth author's name was incorrectly published in the Original Article. The correct author name is Cen Hong.

The Original Article has been corrected.

The original article can be found online at https://doi. org/10.1007/s12325-019-00967-w.

X. Qi $(\bowtie) \cdot$ R. Wang · H. Deng · C. Hong · Q. Li · H. Li · X. Guo $(\bowtie)$

Liver Cirrhosis Study Group, Department of Gastroenterology, General Hospital of Northern Theater Command (Formerly General Hospital of Shenyang Military Area), Shenyang, Liaoning, China

e-mail: xingshunqi@126.com

X. Guo

e-mail: guo_xiao_zhong@126.com

Y. Li

Department of Gastroenterology, The First Hospital of China Medical University, Shenyang, Liaoning, China

L. Lin · M. Wang

Department of Gastroenterology, Shengjing

Hospital, China Medical University, Shenyang,

Liaoning, China

J. Li

Department of Gastroenterology, The First Affiliated Hospital of Jinzhou Medical University, Jinzhou, Liaoning, China
L. Wang

Department of Gastroenterology, Panjin Central Hospital, Panjin, Liaoning, China

S. Zheng

The Sixth People's Hospital of Shenyang, Shenyang, Liaoning, China

Y. Sun

Department of Gastroenterology, Dalian Friendship Hospital of Dalian Medical University, Dalian, Liaoning, China

L. Zhao

Department of Gastroenterology, General Hospital of Liaohe Oilfield Company, Panjin, Liaoning,

China

$\mathrm{X} . \mathrm{Fu}$

Department of Gastroenterology, Ansteel Group Hospital, Anshan, Liaoning, China

X. Qiu

Department of Gastroenterology, General Hospital of Fuxin Mining Industry Group of Liaoning Health Industry Group, Fuxin, Liaoning, China 